\title{
Area postrema syndrome in neuromyelitis optica spectrum disorder: diagnostic challenges and descriptive patterns
}

\author{
Eman M. Khedr ${ }^{\text {* }}$ (D, Hassan M. Farweez ${ }^{1}$, Noha Abo Elfetoh', Eman R. Badawy², Sara Hassanein³, \\ Doaa M. Mahmoud ${ }^{1}$ and Ahmed Nasreldein ${ }^{1}$
}

\begin{abstract}
Background: Although area postrema syndrome (APS) is one of the core clinical features of neuromyelitis optic spectrum disorder (NMOSD), it is frequently misdiagnosed as gastrointestinal or systemic disorders. In this study, we describe the diagnostic challenges in NMOSD patients with APS and their characteristic clinical and radiological features. All patients who attended our university hospitals during the period from March 2019 to August 2020 with a diagnosis of NMOSD according to the latest diagnostic criteria were admitted and evaluated clinically, radiologically with gadolinium-enhanced brain and spinal MRI, measures of serum Anti-Aquaporin 4 (Anti-AQP4) and clinical status using the Expanded Disability Status Scale (EDSS) scores. APS was diagnosed if there was a history of intractable nausea, vomiting, or hiccups (INVH) that had lasted longer than 1 week with the exclusion of other etiologies, or less than $48 \mathrm{~h}$ if associated with a lesion in the dorsal medulla on MRI scan.
\end{abstract}

Results: Twenty out of 90 (22.2\%) identified patients with a diagnosis of NMOSD had a history of unexplained intractable nausea, vomiting or hiccoughs lasting an average of 20 days. Seventeen patients were anti-Aquaporin 4 seropositive. Seven patients (35\%) presented initially with isolated clinical features of APS and were diagnosed only after subsequent relapse. Patients with APS preceding other core clinical presentations (13 cases, 65\%) were diagnosed after development of motor manifestations. All patients developed acute myelitis during the course of illness. Brain and spinal MRI scans showed that 13 had a linear lesion in the dorsal tegmentum of the medulla oblongata adjacent to the fourth ventricle. Otherwise, longitudinally extensive transverse myelitis was found in $80 \%$, while $35 \%$ showed extension of the cord lesion to the AP.

Conclusions: APS as a core clinical characteristic of NMOSD is not a rare presentation as was previously thought and can occur in both AQP4-seropositive and seronegative NMOSD.

Keywords: NMOSD, Area postrema, Area postrema syndrome, AQP4, Vomiting, Hiccoughs

\section{Background}

Neuromyelitis optica spectrum disorder (NMOSD) is an immune-mediated inflammatory disorder of the central nervous system (CNS) that commonly targets the optic nerves and the spinal cord $[1,2]$. After the discovery of

\footnotetext{
*Correspondence: Emankhedr99@yahoo.com

${ }^{1}$ Department of Neurology and Psychiatry, Assiut University,

Assiut 71526, Egypt

Full list of author information is available at the end of the article
}

its specific serological marker (Anti-Aquaporin 4 "AQP4 "), a recent diagnostic criteria has evolved to include the area postrema, the diencephalon, the brainstem and the cerebral hemispheres as other targets for these autoantibodies [3]

Area postrema syndrome (APS) is uncommon neurological presentation characterized by unexplained attacks of intractable nausea, vomiting or hiccoughs (INVH) lasting for more than 1 week (at least $48 \mathrm{~h}$ if associated with a new lesion at dorsal medulla) $[1,4]$. 
The area postrema (AP) is located at the dorsal medulla on the floor of the fourth ventricle, adjacent to the nucleus of the solitary tract (NTS) [5]. It is an area, where the blood-brain barrier (BBB) is more permeable (due to lack of tight junctions between the vascular endothelial cells and the presence of fenestrated capillaries) and aids neurochemical communication and fine control of autonomic functions [6]. The leaky BBB is an entry point for NMO-IgGs, and its enrichment with AQP-4 channels makes the AP an early target in NMOSD [7, 8]. Pathologically, lesions in the AP are characteristically nondestructive with full remission after resolution of inflammation compared to spinal cord or optic nerve lesions [9].

Identification and diagnosis of APS has both diagnostic and prognostic implications. Although nondemyelinating conditions may cause APS [4], when it is accompanied by extension of a spinal cord lesion to the AP together with intractable nausea and vomiting, it is highly specific for AQP4-seropositive NMOSD [10]. More importantly, APS might herald a more severe motor, visual or brainstem lesion [4]. Thus, it is necessary to recognize APS early and to commence therapy as soon as possible to prevent NMOSD recurrence with more devastating neurologic deficits [8].

Unfortunately, when APS occurs at the onset of NMOSD especially in the absence of additional neurological deficits, it is commonly misdiagnosed by gastroenterologists or internists as a digestive system disease or other systemic illness with consequent delay in initiating treatment and risk of exacerbating NMOSD [8].

Most studies of NMOSD have focused on the classic opticospinal presentations, while only few outlined other presentations including APS and were mainly case reports [11-21]. By conducting this study, we aimed to investigate the frequency and diagnostic challenges in NMOSD patients with APS from the south of Egypt with a description of their clinical and radiological features.

\section{Methods}

This was a cross sectional prospective study of all patients who attended Assiut University Hospitals during the period from March 2019 to August 2020 with NMOSD diagnosis according to 2015 IPND diagnostic criteria of NMOSD [1]. All patients with suspected NMOSD during this period were directly interviewed and APS was diagnosed if there was a history of INVH that had lasted longer than 1 week with exclusion of other etiologies or less than $48 \mathrm{~h}$ if associated with a lesion in the dorsal medulla on MRI scan $[1,22]$. NMOSD patients with APS (NMOSD-APS) were admitted and re-evaluated clinically, radiologically and with laboratory analysis.

The following data were obtained:
Clinical data: including age at onset, sex, onset, duration and clinical presentation of APS, type and time of other core clinical features. The number and type of relapse, the initial medical specialty consulted, any misdiagnoses, therapy received and EDSS scores all were recorded.

Radiological data: a new brain and spinal MRI with gadolinium (Gd) was performed for each patient; the previous MRI (when available) was also re-evaluated by an expert neuroradiologist blinded to the clinical presentation. Imaging was performed with 1.5 Tesla MRI (mainly Achieva, Philips Medical Systems, Best, the Netherlands) with protocols of $\mathrm{T} 1$ (with and without contrast) and T2 weighted sequences, fluid-attenuated inversion recovery (FLAIR) in addition to short-tau inversion recovery (STIR) sequences (for spine MRI).

Laboratory data: venous samples were taken from all patients, while in relapse before the start of treatment in plain tubes that were sent to the lab and left to clot for $30 \mathrm{~min}$ at $37^{\circ} \mathrm{c}$ and centrifuged at $3000 \mathrm{rpm}$. The separated serum was used for quantitative determination of Serum AQP4 antibody using Elabscience Biotechnology Inc, Human AQP4 Enzyme-Linked Immunosorbent Assay (ELISA) Kit, USA, Catalog No: E-EL-H0490 according to manufacturer instructions. This ELISA kit used the Sandwich-ELISA technique. The microELISA plate provided in this kit had been pre-coated with an antibody specific to Human AQP-4. Results were recorded as seropositive or seronegative. For the AQP4-seronegative samples, anti-MOG was tested using Elabscience Biotechnology Inc, Human MOG EnzymeLinked Immunosorbent Assay (ELISA) Kit, USA, Cata$\log$ No: E-EL-H0594 using the same technique used for detection of anti-AQP4.

Patients or their family members gave a signed written informed consent after explanation of the study methods was provided. Local ethical committee of Faculty of Medicine has approved the study.

\section{Statistical analysis}

The statistical software SPSS (IBM SPSS Statistics for Windows, Version 24.0. Armonk, NY: IBM Corp.) was used for data processing and analysis. Descriptive analysis, including frequency and percentage was used for the categorical variables. Continuous variables were reported as mean \pm standard deviation (SD) for normally distributed variables and median (interquartile range; IQR) for non-normally distributed variables. Differences between the frequencies of categorical variables were tested with the Pearson Chi-square test or Fisher's exact test; independent $t$ tests were used for continuous variables. $P$ values below 0.05 were considered significant. 


\section{Results}

Twenty out of 90 (22.2\%) identified patients with NMOSD had APS. Table 1 summarizes their demographic, serologic and clinical features. Six patients (30\%) spontaneously gave history of attacks of INVH during the interview, while the remaining fourteen patients (70\%) responded only after applying the direct screening question "have you ever had attacks of vomiting, nausea or hiccoughs lasted at least for 2 days without a known etiology".

All patients consulted initially a gastroenterologist or an internist; all were misdiagnosed with GIT disorders. Six patients out of them (30\%) had objective evidence of weight loss and underwent thorough and even repeated gastroenterological evaluation including abdominal ultrasound and/or CT abdomen, upper endoscopy, as well as several metabolic tests.

All patients had considerable diagnostic delay even after the evolution of neurological deficits. Seven patients (35\%) presented with isolated clinical features of APS and were diagnosed only after subsequent relapse (mean inter-attack duration was $75.7 \pm 57.7$ days ranging from 1 to 6 months). Patients with APS preceding other core clinical presentations within the same attack (13 cases, $65 \%)$ were diagnosed only after they developed additional neurological signs (mean duration of $9.1 \pm 5.2$ days ranging from 4 to 20 days). With emergence of the motor deficits, three cases were initially diagnosed as Guillain Barré syndrome (history of intractable vomiting followed by acute flaccid weakness), while in another 4 patients (20\%) a diagnosis of MS was made before being referred to our center due to overlooked history of APS. None of the cases had received DMT before NMOSD diagnosis.
The mean age at onset of NMOSD-APS patients was $30 \pm 11.3$ years (ranging from 11 to 50 years) similar to the mean age at onset of NMOSD patients without APS $(31.2 \pm 12.0$ years $(6-56))$. There were 16 females and 4 males giving a female to male ratio of 4:1 (compared to 2.8:1 ratio in NMOSD patients without APS). Seventeen patients $(85 \%)$ were AQP4-seropositive and 3 patients were AQP4-seronegative (they tested positive for anti-MOG but a confirmatory testing with a cellbased assay was not available, so they were only considered AQP4-seronegative).

Twelve cases (60\%) had APS at NMOSD-onset, while the other 8 cases (40\%) developed it during subsequent relapses. The average duration of APS was 20 days (IQR) ranging from 7 days to 3 months. All patients presented with nausea and vomiting, while only $6(30 \%)$ had associated hiccoughs. Three patients developed additional respiratory failure requiring mechanical ventilation, Table 2.

The average duration to additional neurological manifestations was 25 days (IQR) (ranging from 4 days to 6 months). Motor sequelae followed APS presentation either within the same attack or in a subsequent relapse (100\% of patients had acute myelitis), while other brain stem involvement and optic neuritis followed in 9 cases (45\%) and 7 cases (35\%), respectively. A significant higher frequency of acute myelitis and brain stem syndrome in NMOSD with APS than without was recorded. During the period of admission, incomplete motor recovery was reported in nine cases $(45 \%)$, no recovery in one case (5\%) and death following extensive brainstem lesion was reported in another case (5\%). Mean EDSS scores were $4.5 \pm 2.6$, Table 2 .

Table 1 Demographic and clinical characteristics of the 20 NMOSD patients with APS compared to NMOSD patients without APS

\begin{tabular}{|c|c|c|c|}
\hline & NMOSD patients with APS $(n=20)$ & $\begin{array}{l}\text { NMOSD patients without APS } \\
(n=70)\end{array}$ & $p$ value \\
\hline Age at onset in years (mean $\pm S D$ ) (Range) & $30 \pm 11.3(11-50)$ & $31.2 \pm 12.0(6-56)$ & 0.440 \\
\hline \multicolumn{4}{|l|}{$\operatorname{Sex}(n, \%)$} \\
\hline Female & $16(80 \%)$ & $52(73.9 \%)$ & \multirow[t]{2}{*}{0.771} \\
\hline Male & $4(20 \%)$ & $18(26.1)$ & \\
\hline \multicolumn{4}{|l|}{ Seroprevalence of Anti-Aquaporin $4(n, \%)$} \\
\hline Aquaporin 4-seropositive & $17(85 \%)$ & $60(85.7 \%)$ & \multirow[t]{2}{*}{1.000} \\
\hline Aquaporin 4-seronegative & $3(15 \%)$ & $10(14.3 \%)$ & \\
\hline \multicolumn{4}{|c|}{ Other core symptoms along the disease course $(n, \%)$} \\
\hline Acute myelitis & $20(100 \%)$ & $51(72.9 \%)$ & 0.005 \\
\hline Optic neuritis & $13(65 \%)$ & $54(77.1 \%)$ & 0.603 \\
\hline Acute brain stem syndrome & $11(55 \%)$ & $20(28.6 \%)$ & 0.028 \\
\hline Acute diencephalic syndrome & $3(15 \%)$ & $3(4.3 \%)$ & 0.121 \\
\hline Acute cerebral syndrome & $5(25 \%)$ & $26(37.1 \%)$ & 0.314 \\
\hline
\end{tabular}


Table 2 Characteristics of APS in NMOSD patients

\begin{tabular}{|c|c|}
\hline \multicolumn{2}{|l|}{ Presentation of the Area postrema syndrome $(n, \%)$} \\
\hline Nausea and vomiting & $14(70 \%)$ \\
\hline Nausea, vomiting and hiccough & $6(30 \%)$ \\
\hline Duration of the Area postrema syndrome in days (IQR) (Range) & $20(7-90)$ \\
\hline Objective weight loss $(n, \%)$ & $6(30 \%)$ \\
\hline Endoscopic maneuver $(n, \%)$ & $6(30 \%)$ \\
\hline \multicolumn{2}{|l|}{ Timing of the Area postrema syndrome attack $(n, \%)$} \\
\hline Initial presentation & $12(60 \%)$ \\
\hline Subsequent attacks & $8(40 \%)$ \\
\hline \multicolumn{2}{|l|}{ Association with other core symptoms ( $n, \%)$} \\
\hline Isolated APS (> 7 days) & $7(35 \%)$ \\
\hline APS followed by another core criteria in the same attack ( $<4$ weeks) & $13(65 \%)$ \\
\hline \multicolumn{2}{|l|}{ Other core symptoms following APS $(n, \%)$} \\
\hline Acute myelitis & $20(100 \%)$ \\
\hline Optic neuritis & $7(35 \%)$ \\
\hline Acute brain stem syndrome & $9(45 \%)$ \\
\hline
\end{tabular}

NMOSD neuromyelitis Optica Spectrum Disorder, APS Area Postrema Syndrome, IQR Interquartile range

A new MRI brain was obtained for all patients with APS; however, only 14 patients had MRI brain during or immediately following APS manifestations. Thirteen of these had a linear lesion in the dorsal tegmentum of the medulla oblongata adjacent to the fourth ventricle in sagittal cuts, while only seven of them (35\%) showed an "inverted V sign" in axial cuts (Fig. 1). Other brain MRI abnormalities included brain stem involvement other than AP (Fig. 2) in 13/20 patients (65\%), periependyma/periventricular lesions in $14 / 20$ patient (70\%), corpus callosum (25\%), optic nerves (20\%), corticospinal tract (15\%), thalamus (15\%), hypothalamus (10\%). Higher frequency of periependyma/periventricular lesions were found in NMOSD patients without APS $(p=0.008)$. Spinal cord MRI obtained during the course of NMOSD showed longitudinally extensive transverse myelitis (LETM) in 16/20 patients (80\%); 7 patients (35\%) showed extension of the cord lesion to the AP (Fig. 3). A summary of the radiological features is given in Table 3.

\section{Discussion}

In the current study, we analyzed the clinical features, radiological characteristics and AQP4-seroprevalence of twenty patients with NMOSD-APS. Although APS is one of the core clinical features of NMOSD along with transverse myelitis (TM) and optic neuritis (ON), its frequency is commonly underestimated, because the nonspecific symptomatology can lead to misdiagnosis by non-neurological specialists even when presenting with other core criteria [20]. The fact that is its frequently reported at disease onset or acute exacerbation, makes it an important warning sign that heralds motor or visual disability or even a life threatening brainstem involvement [4]. Hence it is important to examine clinical and radiological features in such cases with unfamiliar presentations to intervene when necessary to halt devastating sequels.

The main findings of the present study are the high prevalence of APS among the 90 NMOSD diagnosed patients who were directly interviewed. Similar frequencies of APS were reported in a previous Egyptian case series by Salama and colleagues (5 cases, 25\%) [23], in contrast to another recent Egyptian NMOSD study by Kishk and colleagues, in which only 1 case (1.4\%) presented with APS [24]. In fact, the frequency of APS during the disease course varied in different studies from different nations (ranging from as low as $3 \%$ to up to $43 \%$ of NMOSD patients) [11, 21-34]. This can be partially explained by different study designs, although higher frequencies were observed among East Asians and Africans than in other populations suggesting that there may be a genetic influence $[25,35]$.

None of the 20 cases with APS were diagnosed before further serious symptoms became apparent. During episodes of INVH they were misdiagnosed with variable gastrointestinal disorders with several diagnostic procedures that failed to define a local or systemic etiology. Even with emergence of motor manifestations, some patients were initially misdiagnosed. Despite the clinical and radiological overlap between NMOSD and MS, accurate distinction between them is critically important not only for determining appropriate therapy, but also because disease modifying treatment for MS, such as interferon- $\beta$, fingolimod, natalizumab, and alemtuzumab, may aggravate NMOSD $[1,36]$. In a large multicentric 


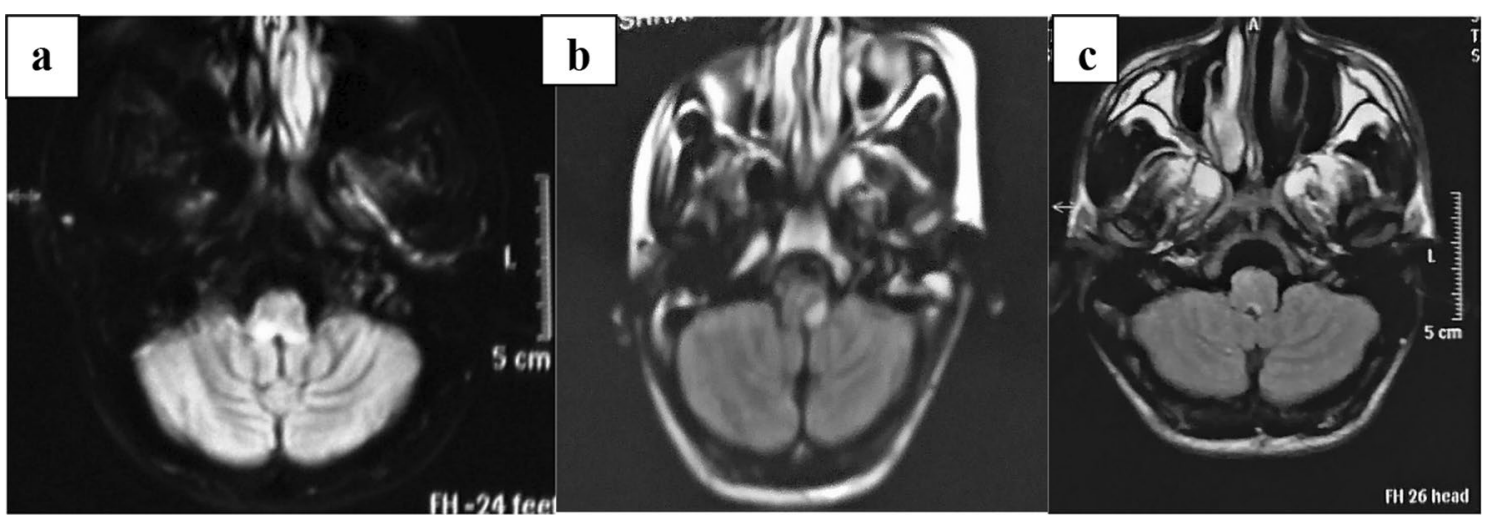

Fig. 1 Axial T2-weighted FLAIR MRI images at the level of the lower medulla oblongata showing hyperintense signals in the area postrema either a bilaterally, $\mathbf{b}$ unilateral (in Left AP), or c periaqueductal (inverted $V$ sign)

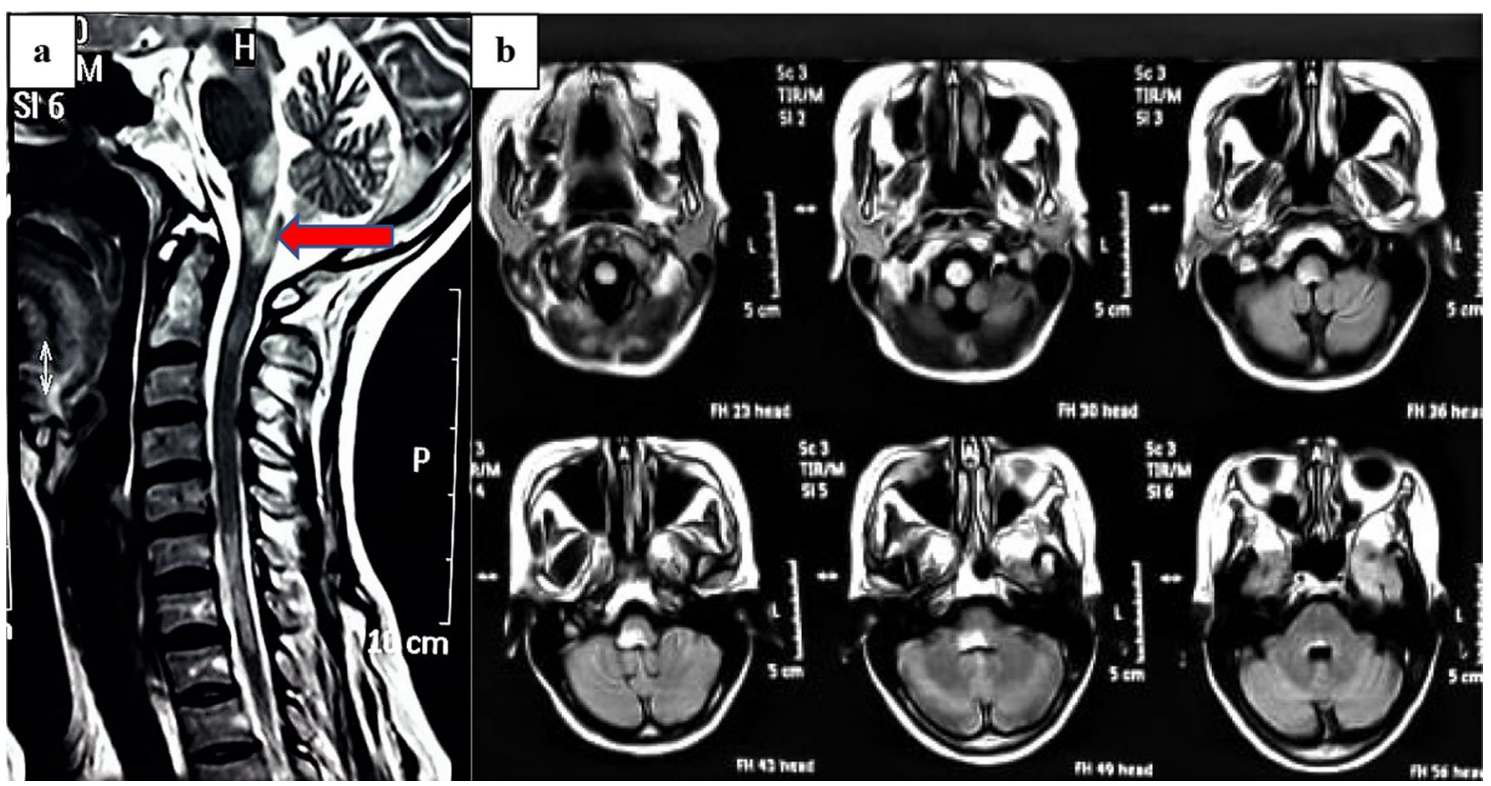

Fig. 2 Brain and cervical MRI of a female patient with fulminant presentation preceded by an APS, with consequent acute respiratory failure, mechanical ventilation, and death from autonomic disturbances. a Sagittal T2-weighted MRI sequence of the cervical cord showing hyperintense lesions in the area postrema (red arrow) on the dorsal inferior surface of the medulla oblongata at the caudal end of the fourth ventricle, extensive brain stem involvement (ventral medulla and pons), two cord lesions (cervical and cervicodorsal). b Axial fluid-attenuated inversion recovery magnetic resonance imaging of the brain showing a hyperintense signal in the medulla oblongata

US study, 29.4\% of NMOSD patients were initially misdiagnosed with MS [37]. Another large study from two centers in Kuwait and Lebanon investigated patients with suspected MS diagnosis and found that $29 \%$ were misdiagnosed of whom 9.5\% turned to have NMOSD [38]

Zhou and colleagues, in a case series of NMOSD patients presenting with APS, suggested that they are predominantly younger with more frequent female involvement. In the current study the slightly higher female to male ratio, however, the same young age at onset as NMOSD patients without APS might be related to the overall younger age at onset of NMOSD in African populations compared to Asians and Europeans which may have a genetic basis [39]. Moreover, as pointed out by Kitley and colleagues, different phenotypes may be due to an age-dependent variability in AQP4 antibody accessibility of the target organs [40], making AP a target in groups with a younger age of onset.

Tendency of APS to occur at disease onset and to precede other core features within the same attack was 
Table 3 Radiological features of the 20 NMOSD patients with APS compared to NMOSD patients without APS

\begin{tabular}{|c|c|c|c|}
\hline MRI brain abnormalities & $\begin{array}{l}\text { NMOSD patients with APS } \\
(n=20)\end{array}$ & $\begin{array}{l}\text { NMOSD patients without APS } \\
(n=70)\end{array}$ & $p$ value \\
\hline Area postrema & $13(65 \%)$ & 0 & 0.000 \\
\hline Linear lesion & $13(65 \%)$ & 0 & \\
\hline Inverted $V$ sign & $7(35 \%)$ & 0 & \\
\hline Other brain stem structures & $13(65 \%)$ & $25(44.6 \%)$ & 0.192 \\
\hline Periependymal/Periventricular & $14(70 \%)$ & $53(94.6 \%)$ & 0.008 \\
\hline Corpus callosum & $5(25)$ & $17(30.4 \%)$ & 0.778 \\
\hline Corticospinal tract & $3(15 \%)$ & $5(8.9 \%)$ & 0.427 \\
\hline Thalamus & $3(15 \%)$ & $13(23.2 \%)$ & 0.537 \\
\hline Hypothalamus & $2(10 \%)$ & $9(16.1 \%)$ & 0.717 \\
\hline \multicolumn{4}{|l|}{ Abnormal MRI spinal cord } \\
\hline $\begin{array}{l}\text { Longitudinally extensive transverse myelitis (> } 3 \text { seg- } \\
\text { ments) (LETM) }\end{array}$ & $16(80 \%)$ & $30(57.7 \%)$ & 0.103 \\
\hline Short segment transverse myelitis (STM) & $4(20 \%)$ & $22(42.3 \%)$ & 0.103 \\
\hline Extension to medulla and Area postrema & $7(35 \%)$ & 0 & $<0.0001$ \\
\hline \multicolumn{4}{|l|}{ Site } \\
\hline Cranio-cervical & $7(35 \%)$ & $3(6 \%)$ & 0.004 \\
\hline Cervical & $5(25 \%)$ & $20(40 \%)$ & 0.167 \\
\hline Cervico-dorsal & $5(25 \%)$ & 16 (32\%) & 0.73 \\
\hline Others & $3(15 \%)$ & $11(22 \%)$ & 0.270 \\
\hline
\end{tabular}

The bold values denotes the statistical significant values

LETM longitudinally extensive transverse myelitis, STM Short segment transverse myelitis

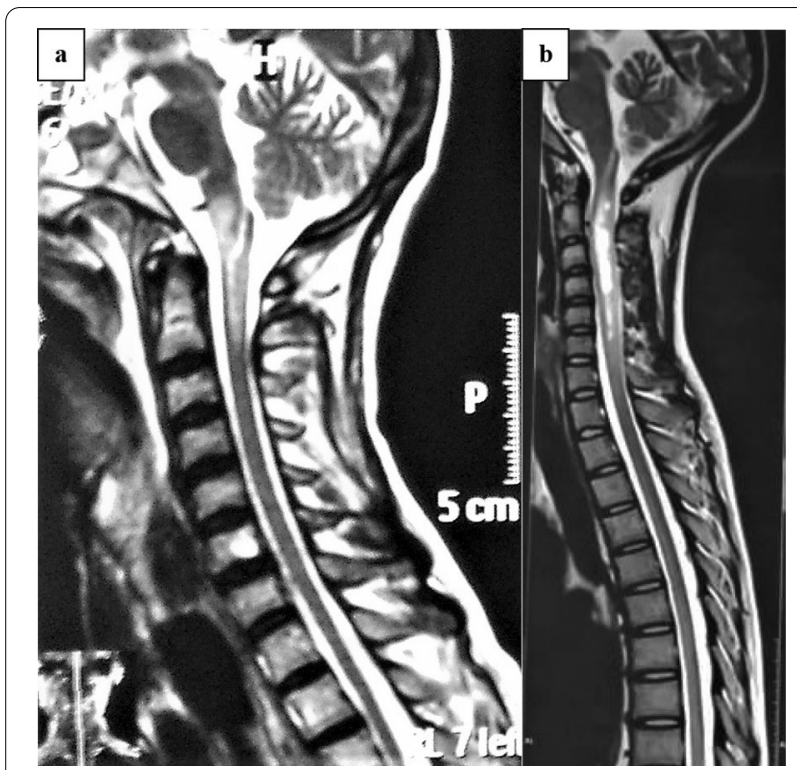

Fig. $3 \mathrm{MRI}$ of the cervical spine T2WI sequence demonstrating T2 hyperintensity at the area postrema: $\mathbf{a}$ area postrema lesion with involvement of the medulla oblongata and the upper cervical cord. $\mathbf{b}$ longitudinally extensive transverse myelitis (LETM) with bright spotty lesions extending up to the area postrema and down to C6

evident in the current study. In agreement with a large international multicentric study by Shosha and colleagues, where $58-68 \%$ of APS attacks were found to precede acute myelitis or optic neuritis [22]. This could be explained by the specific anatomical features of AP facilitating the entry of the pathogenic NMO-IgGs hence the earlier involvement before other core clinical features $[7,8,27]$. Thus, early identification and initiation of immunosuppressive therapy would prevent the development of more devastating neurologic deficits [32].

Although cases with hiccoughs in the context of APS are less frequently reported as in our cases compared to nausea and vomiting [22], no peculiar features of the hiccough attacks could be found upon reviewing literature [32]. Much less frequently reported was cardiac arrest and acute respiratory failure with APS syndrome due to the connection with NTS $[7,8,39,41,42]$.

Acute myelitis was the most common subsequent event in all patients, this was consistent with several studies, as reported by Shosha and colleagues [22] and Zhou and colleagues [39] confirming the spinal cord as being the most frequent target after an episode of APS and emphasize the need for urgent implementation of immunotherapy as soon as APS is recognized as a part of NMOSD.

A lesion in the dorsal brainstem adjacent to the fourth ventricle including the AP and the nucleus of the solitary tract is one of the most specific brain MRI abnormalities corresponds to APS and reported in $7 \%$ to $46 \%$ of patients with NMOSD $[43,44]$. These lesions may appear 
as linear FLAIR/T2-weighted hyperintensities of the dorsomedial structures in sagittal cuts which may extend to involve the central canal [43]. While axial cuts may show an "inverted V sign" corresponding to the symmetrical involvement of the AP located on both sides along the floor of the fourth ventricle and connected together at the midline [39].

Due to the non-destructive nature of the lesions at AP and brainstem, delayed MRI after remission of APS or initiation of treatment might miss an AP lesion without evidence in subsequent imaging [6]. Li and colleagues reported similar observations, where 12 of their patients with no MRI during their acute attack of APS, showed no AP lesions during follow-up. In addition, they reported absence of AP lesion in another 5 patients at presentation suggesting the possibility that brain imaging may have missed evolving lesions or that the lesions were too small to be detected by MRI [11]. This emphasizes the primary importance of clinical evidence and the need to consider APS even with a negative radiology.

Another interesting finding was the presence of significantly higher levels of periepedymal/periventricular lesions in NMOSD patients without APS compared to NMOSD-APS, which might suggest a different point of entry of the pathogenic autoantibodies or a different distribution of the target channels [45].

Several prior studies have suggested spinal cord lesions extending to the AP (evident in nearly $1 / 3$ of our patients) are specific for NMOSD $[1,46]$. However, Dubey and colleagues reported that it is more specific for AQP4-IgG positive NMOSD when INVH accompanies the extension of a spinal cord lesion to the AP, therefore, supporting the importance of clinical evidence rather than the isolated radiology to identify NMOSD-APS [10].

On the other hand, although APS is considered highly specific for AQP4-seropositivity [14, 19, 32, 46-48] APS in AQP4-seronegative patients (including patients with anti-Myeline oligodendrocyte glycoprotein "MOG"IgGs) are being increasingly recognized [39]. Three AQP4-seronegative cases were identified in the present study (15\%) and tested positive for anti-MOG. The fact that the lack of BBB at the AP allows entry of other pathogenic autoantibodies making it likely that AP may be involved in AQP4-seronegative NMOSD [49, 50].

Adding more challenges to diagnosis, a false negative AQP4-test might be related to factors other than the type of Assay used including timing of sampling (higher titers during relapse and lower titers during remission or after initiation of immunotherapy) [51]. Recently, Akaishi and colleagues and $\mathrm{Li}$ and colleagues suggested that AQP4-IgG titers during the first attack were lower in NMOSD-APS compared to NMOSD patients with optic neuritis, implying that there may be a particular pathogenic process related to AP that needs further research $[11,52]$. Rattanathamsakul, and colleagues also reported that APS was twice as common in AQP4-IgG negative NMOSD patients both at initial presentation and thereafter [34]. Therefore, NMOSD should be considered in context of strong clinical suspicion even with negative serological markers.

\section{Limitations}

Certain challenges and limitations need to be acknowledged in the current study, particularly the unavailability of the cell-based assays (CBA) for both AQP4 and anti-MOG. However, this study might encourage future studies with Anti-MOG testing using CBA to accurately elucidate the frequency of MOG-seropositive cases with APS.

Future research: Since oxidative stress plays a crucial role in the physiopathology of neuroimmunological diseases measures of thiol homeostasis might be useful for monitoring disease activity in future studies. Indeed, a timely diagnosis of APS is crucial for the initiation of specific therapies positively affecting the outcome.

\section{Conclusions}

APS as a core clinical characteristic of NMOSD is not a rare presentation as was previously thought and can occur in both AQP4 and MOG-seropositive patients. The clinical features of INVH after exclusion of alternative diagnoses are the most important diagnostic tool.

\section{Abbreviations \\ AP: Area postrema; APS: Area postrema syndrome; AQP-4: Aquaporin 4; BBB: Blood-brain barrier; CNS: Central nervous system; CBA: Cell-based assay; EDSS: Expanded disability status score; ELISA: Enzyme-Linked Immunosorbent Assay; FLAIR: Fluid-attenuated inversion recovery; Gd: Gadolinium; INVH: Intractable nausea, vomiting or hiccoughs; IQR: Interquartile range; MRI: Magnetic reso- nance imaging; NMOSD: Neuromyelitis optic spectrum disorder; NMOSD-APS: Neuromyelitis optic spectrum disorder with area postrema syndrome; NTS: Nucleus of the solitary tract; STIR: Short-tau inversion recovery.}

\section{Acknowledgements}

Not applicable

\section{Authors' contributions}

EMK: contributed to conceptualization; project administration; supervision; manuscript writing with input from all authors. HMF: contributed to research idea development and supervised the findings of the work. NA: contributed to clinical investigation and clinical data analysis. AN: contributed to clinical investigation and clinical data analysis. DMM: contributed to collecting data, clinical investigation, statistical analysis and assisted in original draft writing. ERB: performed and analyzed laboratory investigations. SH: analyzed radiological data. All authors read and approved the final manuscript.

\section{Funding}

This work was supported by a grant from the Grants Office, Faculty of Medicine, Assiut University, Assiut, Egypt (2019-01-27-001).

\section{Availability of data and materials}

The data sets used and/or analyzed during the current study are available from the corresponding author on reasonable request. 


\section{Declarations}

\section{Ethics approval and consent to participate}

Patients or their family members gave signed an informed written consent after explanation of the study methods was provided. Local ethical committee of Faculty of Medicine, Assiut University has approved the study (IRB no: 17200291).

\section{Consent for publication}

Not applicable.

\section{Competing interests}

The authors declare that they have no competing interests.

\section{Author details}

'Department of Neurology and Psychiatry, Assiut University, Assiut 71526, Egypt. ${ }^{2}$ Department of Clinical Pathology, Assiut University, Assiut, Egypt. ${ }^{3}$ Department of Radiology, Assiut University, Assiut, Egypt.

Received: 11 August 2021 Accepted: 25 September 2021 Published online: 12 November 2021

\section{References}

1. Wingerchuk DDM, Banwell B, Bennett JJL, Cabre P, Carroll W, Chitnis T, et al. International consensus diagnostic criteria for neuromyelitis optica spectrum disorders. Neurology. 2015:85(2):177-89.

2. Cañellas AR, Gols AR, Izquierdo JR, Subirana MT, Gairin XM. Idiopathic inflammatory-demyelinating diseases of the central nervous system. Neuroradiology. 2007. https://doi.org/10.1007/s00234-007-0216-2.

3. Bennett JL. Finding NMO: The evolving diagnostic criteria of neuromyelitis optica. J Neuro-Ophthalmology. 2016;36(3):238-45.

4. Camara-Lemarroy CR, Burton JM. Area postrema syndrome: A short history of a pearl in demyelinating diseases. Mult Scler J. 2019;25(3):325-9

5. DE SOUZA TFS. A concise historical perspective of the area postrema structure and function. Arq Neuropsiquiatr. 2020;78(2):121-3.

6. Lucchinetti CF, Guo Y, Popescu C, Fujihara K, Itoyama Y, Misu T. The pathology of an autoimmune astrocytopathy: lessons learned from neuromyelitis optica. Brain Pathol. 2014;24(1):83-97.

7. Price CJ, Hoyda TD, Ferguson AV, et al. The area postrema: a brain monitor and integrator of systemic autonomic state. Neuroscientist. 2008;14(2):182-94.

8. Okada K, Kobata M, Naruke S. Neuromyelitis optica spectrum disorder with area postrema syndrome. Neurol Clin Pract. 2019;9(2):173-5. https:// www.ncbi.nlm.nih.gov/pmc/articles/PMC6461417/.

9. Roemer SF, Parisi JE, Lennon VA, Benarroch EE, Lassmann H, Bruck W, et al. Pattern-specific loss of aquaporin-4 immunoreactivity distinguishes neuromyelitis optica from multiple sclerosis. Brain. 2007;130(5):1 194-205.

10. Dubey D, Pittock SJ, Krecke KN, Flanagan EP. Association of extension of cervical cord lesion and area postrema syndrome with neuromyelitis optica spectrum disorder. JAMA Neurol. 2017;74(3):359-61. https://jaman etwork.com/journals/jamaneurology/fullarticle/2596240.

11. Li R, Lu D, Li H, Wang Y, Shu Y, Chang Y, et al. Neuromyelitis optica spectrum disorders with non opticospinal manifestations as initial symptoms: a long-term observational study. BMC Neurol. 2021;21(1):1-10.

12. Treviño-Frenk I, Dominguez-Moreno R, Tristan-Samaniego DP, Davila-Sosa D, Barriga-Maldonado GV. Area postrema syndrome as a debut of neuromyelitis optica spectrum disorders. Neurol Clin Neurosci. 2019;7(5):301-2.

13. Apiwattanakul M, Popescu BF, Matiello M, Weinshenker BG, Lucchinetti CF, Lennon VA, et al. Intractable vomiting as the initial presentation of neuromyelitis optica. Ann Neurol. 2010;68(5):757-61. https://pubmed. ncbi.nlm.nih.gov/21031587/.

14. Dandu VH, Siddamreddy S, Meegada S, Muppidi V, Challa T. Isolated area postrema syndrome presenting as intractable nausea and vomiting. Cureus. 2020;12(2):1-9.

15. Takahashi Y, Manabe Y, Nakano Y, Yunoki T, Kono S, Narai H, et al. Fulminant case of neuromyelitis optica spectrum disorder initiated with area postrema symptoms. Neurol Clin Neurosci. 2016;4(3):121-3.
16. Chan KH, Vorobeychik G. Area postrema syndrome: A neurological presentation of nausea, vomiting and hiccups. BMJ Case Rep. 2020:13(11):238588

17. Schattner A, Karni A. Intractable vomiting and the medulla: Neuromyelitis optica spectrum disorder presenting as area postrema syndrome. Postgrad Med J. 2018;94(1118):724.

18. Popescu BFG, Lennon VA, Parisi JE, Howe CL, Weigand SD, Cabrera-Gómez JA, et al. Neuromyelitis optica unique area postrema lesions: Nausea, vomiting, and pathogenic implications. Neurology. 2011;76(14):1229-37.

19. Snyder A, Smedley AD, Reich SG. Intractable nausea due to the area postrema syndrome of neuromyelitis optica: an uncommon cause of a common symptom. J Emerg Med. 2017;53(5):e73-6. https://doi.org/10. 1016/j.jemermed.2017.06.031.

20. Misu T, Fujihara K, Nakashima I, Sato S, Itoyama Y. Intractable hiccup and nausea with periaqueductal lesions in neuromyelitis optica. Neurology. 2005:65(9):1479-82.

21. Mandaliya R, Boigon M, Smith DG, Bhutani S, Ali N, Hilton C, et al. A diagnostic challenge in a young woman with intractable hiccups and vomiting: a case of neuromyelitis optica. J Community Hosp Intern Med Perspect. 2015:5(5):28850

22. Shosha E, Dubey D, Palace J, Nakashima I, Jacob A, Fujihara K, et al. Area postrema syndrome: Frequency, criteria, and severity in AQP4-lgG-positive NMOSD. Neurology. 2018;91(17):E1642-51.

23. Salama S, Marouf H, Ihab Reda M, Mansour AR, ELKholy O, Levy M. Clinical and radiological characteristics of neuromyelitis optica spectrum disorder in the North Egyptian Nile Delta. J Neuroimmunol. 2018. https://doi.org/ 10.1016/i.jneuroim.2018.08.014.

24. Kishk NA, Abdelfattah W, Shalaby NM, Shehata HS, Hassan A, Hegazy MI, et al. The aquaporin4-lgG status and how it affects the clinical features and treatment response in NMOSD patients in Egypt. BMC Neurol. 2021;21(1):1-8.

25. Kim SH, Mealy MA, Levy M, Schmidt F, Ruprecht K, Paul F, et al. Racial differences in neuromyelitis optica spectrum disorder. Neurology. 2018;91(22):E2089-99.

26. Bennis A, El Otmani H, Benkirane N, Harrizi I, El Moutawakil B, Rafai MA, et al. Clinical course of neuromyelitis optica spectrum disorder in a moroccan cohort. Mult Scler Relat Disord. 2019;30(February):141-8. https://doi.org/10.1016/j.msard.2019.02.012.

27. Takahashi T, Miyazawa I, Misu T, Takano R, Nakashima I, Fujihara K, et al. Intractable hiccup and nausea in neuromyelitis optica with anti-aquaporin-4 antibody: A herald of acute exacerbations. J Neurol Neurosurg Psychiatry. 2008:79(9):1075-8

28. Ahmed SF, Al-Hashel JY, Behbehani R, Lamdhade S, Alroughani R. Epidemiology of neuromyelitis optica spectrum disorders patients in Kuwait. Mult Scler Relat Disord. 2020;37:101555.

29. Shosha E, Al Asmi A, Nasim E, Inshasi J, Abdulla F, Al Malik Y, et al. Neuromyelitis optica spectrum disorders in Arabian Gulf (NMOAG); establishment and initial characterization of a patient registry. Mult Scler Relat Disord. 2020. https://doi.org/10.1016/j.msard.2019.101448.

30. Correa Diaz EP, Vasconez V, Paredes VE, Arroyo HH, Morales DC. Neuromyelitis optica spectrum disorder in Quito. Ecuador Mult Scler. 2017:23:74. https://doi.org/10.1177/1352458517693959.

31. Edgar Patricio CD, Eleanor THG, Francisco José CZ, Gabriela ACM, Hyland $A O$, Fernando $G L$, et al. Clinical and radiological profile of neuromyelitis optica spectrum disorders in an Ecuadorian cohort. Mult Scler Relat Disord. 2020;44:102208. https://doi.org/10.1016/j.msard.2020.102208.

32. Iorio R, Lucchinetti CF, Lennon VA, Farrugia G, Pasricha PJ, Weinshenker $B G$, et al. Intractable nausea and vomiting from autoantibodies against a brain water channel. Clin Gastroenterol Hepatol. 2013;11(3):240-5.

33. Pittock SJ, Weinshenker BG, Lucchinetti CF, Wingerchuk DM, Corboy JR, Lennon VA. Neuromyelitis optica brain lesions localized at sites of high aquaporin 4 expression. Arch Neurol. 2006;63(7):964-8.

34. Rattanathamsakul N, Kaveeta C, Siritho S, Thakolwiboon S, Prayoonwiwat $\mathrm{N}$. The 2015 IPND criteria increases the yield in diagnosis of neuromyelitis optica spectrum disorder in thai patients compared to the 2006 diagnostic criteria. Mult Scler Relat Disord. 2020:43:102218.

35. Shosha E, Majed M, Weinshenker B, Flanagan E, Huebert C, Sagen J, et al. Defining area postrema (AP) attacks in autoimmune AQP4 channelopathies/neuromyelitis optica spectrum disorders (NMOSD) (P6.386). Neurology. 2017:88(16 Supplement):P6.386. 
36. Jarius S, Paul F, Aktas O, Asgari N, Dale RC, De Seze J, et al. Mog encephalomyelitis: International recommendations on diagnosis and antibody testing. Nervenarzt. 2018;89(12):1388-99.

37. Mealy MA, Wingerchuk DM, Greenberg BM, Levy M. Epidemiology of neuromyelitis optica in the United States: a multicenter analysis. Arch Neurol. 2012;69(9):1176-80. https://doi.org/10.1001/archneurol.2012.314.

38. Yamout BI, Khoury SJ, Ayyoubi N, Doumiati H, Fakhreddine M, Ahmed SF, et al. Alternative diagnoses in patients referred to specialized centers for suspected MS. Mult Scler Relat Disord. 2017;18:85-9.

39. Zhou C, Liao L, Sun R, Wang J, Di W, Zhu Y, et al. Area postrema syndrome as initial manifestation in neuromyelitis optica spectrum disorder patients: a retrospective study. Rev Neurol (Paris). 2021;177(4):400-6.

40. Kitley J, Leite MI, Nakashima I, Waters P, McNeillis B, Brown R, et al. Prognostic factors and disease course in aquaporin-4 antibody-positive patients with neuromyelitis optica spectrum disorder from the United Kingdom and Japan. Brain. 2012;135(6):1834-49.

41. Hor JY, Asgari N, Nakashima I, Broadley SA, Leite MI, Kissani N, et al. Epidemiology of neuromyelitis optica spectrum disorder and its prevalence and incidence worldwide. Front Neurol. 2020;11:1-13. https://www.front iersin.org/article/10.3389/fneur.2020.00501/full.

42. Okada S, Takarabe S, Nogawa S, Abe T, Morishita T, Mori M, et al. Persistent hiccups followed by cardiorespiratory arrest. Lancet. 2012;380(9851):1444.

43. Kim HJ, Paul F, Lana-Peixoto MA, Tenembaum S, Asgari N, Palace J, et al. MRI characteristics of neuromyelitis optica spectrum disorder: an international update. Neurology. 2015;84(11):1165-73.

44. Dutra BG, da Rocha AJ, Nunes RH, Maia AC, et al. Neuromyelitis optica spectrum disorders: spectrum of MR imaging findings and their differential diagnosis. Radiographics. 2018;38(1):169-93.

45. Matiello M, Schaefer-Klein J, Sun D, Weinshenker BG. Aquaporin 4 expression and tissue susceptibility to neuromyelitis optica. JAMA Neurol. 2013;70(9):1118-25
46. Iorio R, Damato V Mirabella M, Evoli A, Marti A, Plantone D et al Distinctive clinical and neuroimaging characteristics of longitudinally extensive transverse myelitis associated with aquaporin-4 autoantibodies. J Neurol. 2013;260(9):2396-402.

47. Tan CT. International consensus diagnostic criteria for neuromyelitis optica spectrum disorders. Neurology. 2016;86(5):491-2.

48. Hyun JW, Kwon YN, Kim SM, Lee HL, Jeong WK, Lee HJ, et al. Value of Area Postrema Syndrome in Differentiating Adults With AQP4 vs. MOG Antibodies Front Neurol. 2020;11(June):1-4.

49. Kunchok A, Krecke KN, Flanagan EP, Jitprapaikulsan J, Lopez-Chiriboga AS, Chen JJ, et al. Does area postrema syndrome occur in myelin oligodendrocyte glycoprotein-lgG-associated disorders (MOGAD)? Neurology. 2020;94(2):85-8.

50. Jarius S, Kleiter I, Ruprecht K, Asgari N, Pitarokoili K, Borisow N, et al. MOG-IgG in NMO and related disorders: a multicenter study of 50 patients. Part 3: Brainstem involvement-frequency, presentation and outcome. J Neuroinflammation. 2016;13(1):281. https://doi.org/10.1186/ s12974-016-0719-z.

51. Alroughani R, Qadi N, Inshasi J, Shosha E. Neuromyelitis optica spectrum disorders in the Arabian Gulf: challenges and growing experience. Mult Scler J - Exp Transl Clin. 2020;6(1):205521731985019.

52. Akaishi T, Takahashi T, Himori N, Fujihara K, Misu T, Abe M, et al. Serum AQP4-lgG level is associated with the phenotype of the first attack in neuromyelitis optica spectrum disorders. J Neuroimmunol [Internet]. 2020;340:577168

\section{Publisher's Note}

Springer Nature remains neutral with regard to jurisdictional claims in published maps and institutional affiliations.

\section{Submit your manuscript to a SpringerOpen ${ }^{\circ}$ journal and benefit from:}

- Convenient online submission

- Rigorous peer review

- Open access: articles freely available online

- High visibility within the field

- Retaining the copyright to your article

Submit your next manuscript at $\boldsymbol{\nabla}$ springeropen.com 